\title{
Long lifetime polarized electron beam production from negative electron affinity GaAs activated with Sb-Cs-O: Trade-offs between efficiency, spin polarization, and lifetime
}

\author{
Luca Cultrera๑, Alice Galdi®, Jai Kwan Bae®, Frank Ikponmwen, \\ Jared Maxson, and Ivan Bazarov \\ Cornell Laboratory for Accelerator-Based Sciences and Education, Cornell University, \\ Ithaca, New York 14853, USA
}

(Received 24 July 2019; accepted 13 January 2020; published 10 February 2020)

\begin{abstract}
GaAs-based photocathodes are the state-of-the-art in the production of highly spin-polarized electron beams for accelerator and microscopy applications. While various novel structures of GaAs have been shown to increase the degree of polarization and quantum efficiency, all GaAs-based photocathodes require activation to negative electron affinity (NEA) to operate at the photon energies where the highest spin polarization is achieved. In this work, we report on NEA activation of bulk GaAs performed using Sb-Cs$\mathrm{O}$. We show this activation layer to be more robust with respect to traditional Cs and O layer in terms of charge extraction lifetime. This new activation layer is shown to improve the dark lifetime up to one order of magnitude and the charge extraction lifetime up to a factor of about 60 , when compared with the traditional Cs-O activating layer. Trade-offs with other relevant parameters like quantum efficiency and electron spin polarization are also discussed.
\end{abstract}

DOI: 10.1103/PhysRevAccelBeams.23.023401

\section{INTRODUCTION}

Photocathodes that produce highly spin polarized electron beams play a critical role in current and future nuclear and high energy physics facilities, such as the Electron Ion Collider and the International Linear Collider [1-3]. Additionally, spin polarized electron sources are critical for various forms of electron microscopy capable of probing the magnetic properties of materials $[4,5]$. GaAs superlattice (SL) based photocathodes are generally considered the best option for spin polarized electron sources: advanced band structure engineering has enabled photocathode structures capable of providing larger than $80 \%$ spin polarization and quantum efficiency (QE) of a few percents at the same wavelength [6]. The operation of all photocathodes based on GaAs with photon energy near the band gap energy is possible only by activation to negative electron affinity (NEA) conditions, which is achieved at the vacuum interface of the photocathode upon exposure to Cs and oxygen or $\mathrm{NF}_{3}[7,8]$. Unfortunately, the poor stability of this NEA activating layer determines the relatively short operational lifetime of GaAs based photocathodes and

*1c572@cornell.edu

Published by the American Physical Society under the terms of the Creative Commons Attribution 4.0 International license. Further distribution of this work must maintain attribution to the author(s) and the published article's title, journal citation, and DOI. remains one of the primary limits for applications requiring high average current electron beams for prolonged period of time, with examples including nuclear physics experiments such as the production of polarized positrons [9]. Beyond polarized applications, the short lifetime of GaAs photocathodes has prevented its use in high brightness applications like $\mathrm{x}$-ray free electron lasers, even though cesiated GaAs generates a low intrinsic emittance beam with a high QE [10].

The use of heterojunction photocathodes based on GaAs and very thin layers of alkali antimonides was proposed several years ago: experimental results showed that with a proper choice of band alignments a more robust NEA surface can be formed at the expense of a low QE [11-17].

Typically not all the properties of a photocathode $(\mathrm{QE}$, intrinsic emittance, response time, lifetime) can be optimized simultaneously and thus it is important to characterize the potential trade-offs that exist among these parameters. In this work, we report on the preparation and characterization of photocathodes based on bulk GaAs activated to NEA using $\mathrm{Sb}$, Cs, and oxygen. We explore the trade-offs between the spectral response, spin polarization, and lifetime of these cathodes. To compare the multiple samples studied, we also propose a figure of merit for spin polarized photoelectron sources that includes the lifetime.

Among the common alkali antimonides photocathodes, $\mathrm{Cs}_{3} \mathrm{Sb}$ is interesting for this purpose because of its band gap of $1.6 \mathrm{eV}$, which is larger than that of GaAs, and its relatively small electron affinity (about $0.45 \mathrm{eV}$ [18]). 


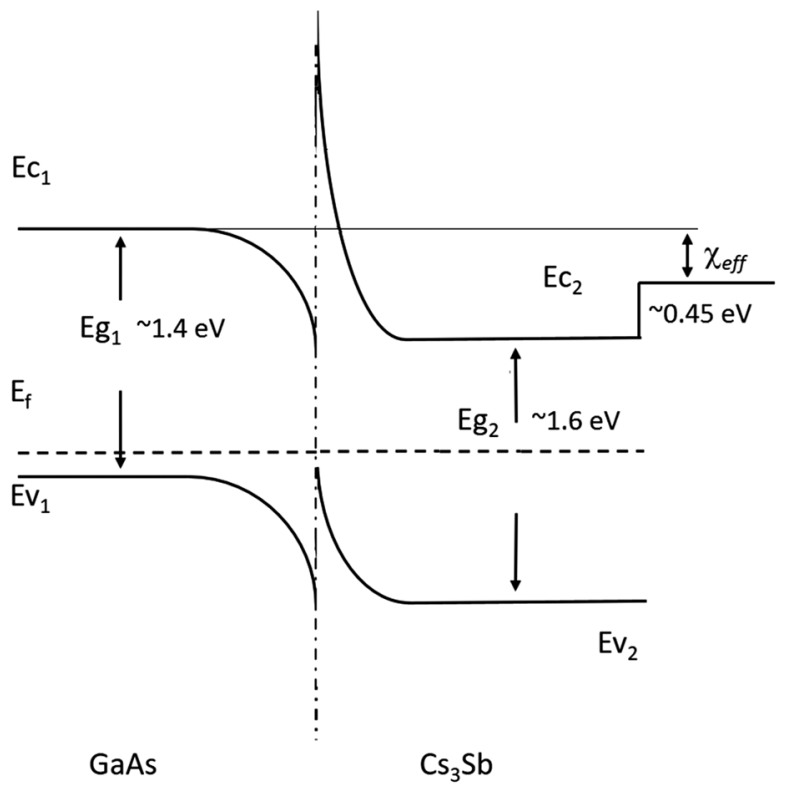

FIG. 1. Energy band diagram of GaAs with a $\mathrm{Cs}_{3} \mathrm{Sb}$ activation layer. When photon energies lower than $1.6 \mathrm{eV}$ (about $780 \mathrm{~nm}$ ) illuminate the sample, photoelectrons should be excited only from the GaAs conduction band.

While it is known that the doping of the $\mathrm{Cs}_{3} \mathrm{Sb}$ can be tuned from p-type to n-type by properly dosing Cs during the growth [19], the combination of its band gap and electron affinity suggests that it may provide NEA on GaAs as an intrinsic semiconductor. This is illustrated in the band diagram in Fig. 1.

If the NEA can be achieved with a very thin layer of $\mathrm{Cs}_{3} \mathrm{Sb}$, it should be possible to primarily excite electrons to the conduction band of GaAs with minimal light absorption and electron excitation in the $\mathrm{Cs}_{3} \mathrm{Sb}$ region, especially in the near infrared spectral region where the spin polarization of electrons in GaAs is maximal.

The role played by electron transport at the interface of such heterostructures can dramatically affect photoemission performance. In the case of GaAs activated with alkali antimonides, the quantum mechanical reflection at the heterojunction is one of the key factors limiting the $\mathrm{QE}$ [13]. While modeling the tunneling across the interface is beyond the scope of this work, recent experimental results [20] in the NEA activation with $\mathrm{Cs}_{3} \mathrm{Sb}$ pointed out that in order to achieve a large QEs in the IR, the surface of GaAs must be initially fully activated to NEA using Cs and oxygen. This suggests that the shape of the potential barrier at the interface between $\mathrm{GaAs}$ and $\mathrm{Cs}_{3} \mathrm{Sb}$ may be strongly influenced by a segregated Cs-O dipole layer and that the dipole layer plays a critical role in the photoelectron transport.

\section{EXPERIMENTAL RESULTS}

Experiments have been conducted using a cluster of interconnected UHV chambers hosted by the photocathode laboratory at Cornell University. Here, samples can be transferred from a vacuum chamber to another in vacuum levels better than $1 \times 10^{-10}$ Torr using magnetically coupled manipulators without being exposed to air. A schematics of the vacuum chambers arrangement is reported in Fig. 2.

Several highly $p$-doped $\left(\mathrm{Zn} 5 \times 10^{18} \mathrm{~cm}^{-3}\right)$ GaAs (100) specimens were cleaved from the same wafer. Surface cleaning was performed with isopropyl alcohol, demineralized water, and wet-etching with $1 \% \mathrm{HF}$ for $30 \mathrm{~s}$ to remove the native oxide layer [21]. Samples were then rinsed again with demineralized water, dried with nitrogen gas, and either connected to the holder and moved into vacuum or bagged in a dry nitrogen atmosphere. One of the GaAs samples was activated to NEA using Cs and oxygen and is used as a reference. All the other samples were activated to NEA using the procedure described below.

In the bialkali growth vacuum chamber (see Fig. 2), the samples were heat cleaned at $500{ }^{\circ} \mathrm{C}$ for about 10 hours to ensure that the surface was free from contaminants. Then, the temperature was lowered to about $120^{\circ} \mathrm{C}$ and held constant until the end of the growth. Samples were illuminated with the light of a $780 \mathrm{~nm}$ continuous wave laser diode modulated by a mechanical chopper and held in vacuum on an electrically insulated holder. A $-18 \mathrm{~V}$ bias was applied to the samples, and the photocurrent was measured using a lock-in amplifier.

The typical sequence of steps in the activation of the samples with Sb-Cs-O is the following (see Fig. 3). After the heat cleaning cycle, the sample is cooled by lowering the heater current. Because of the Cs vapors already present inside the growth chamber, we usually found samples already capable of photoemitting electrons at $780 \mathrm{~nm}$, indicating some initial Cs coverage of the GaAs. As the Cs and $\mathrm{Sb}$ effusion cells are heated to reach the respective operating temperatures of about $65^{\circ} \mathrm{C}$ and $440^{\circ} \mathrm{C}$ we usually observe an additional increase in the photocurrent intensity. We believe that this is due to the fact that a small

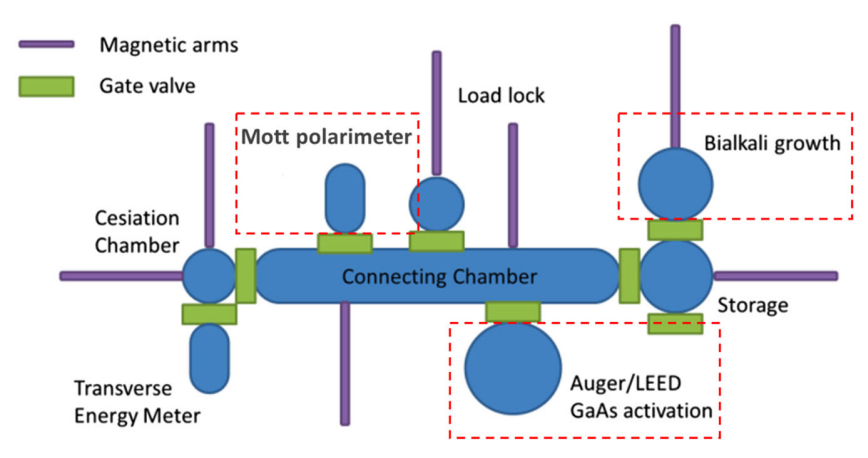

FIG. 2. Schematic of the cluster of vacuum chambers in the photocathode laboratory at Cornell University. Samples are transferred from one chamber to another in a vacuum level better than $1 \times 10^{-10}$ Torr. The experimental chambers used in this set of experiments are indicated with dotted red lines. 


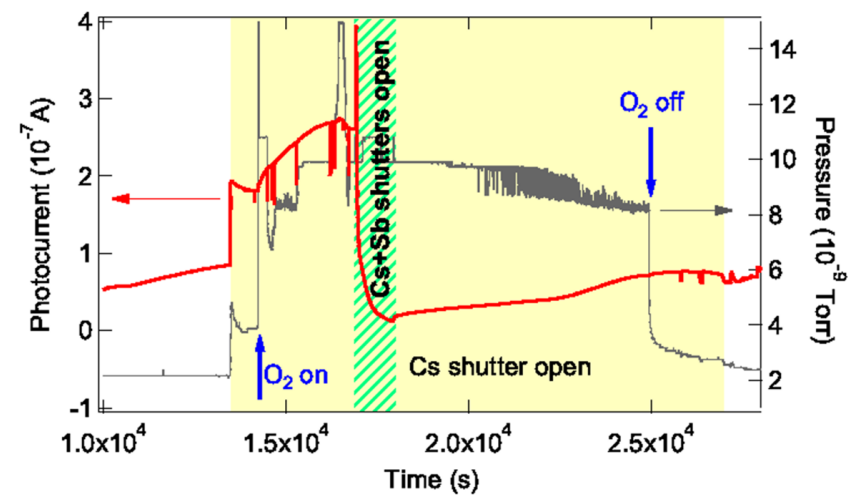

FIG. 3. Typical growth parameters of a Cs-Sb-O coated GaAs sample. Left axis: Photocurrent; the shaded area represents the time interval where the Cs shutter was kept open, the striped area is when both $\mathrm{Cs}$ and $\mathrm{Sb}$ were codeposited. Right axis: Total pressure during deposition. The base pressure before opening the shutters and the oxygen valve was $2.2 \times 10^{-9}$ Torr.

quantity of Cs can still escape from the effusion cell even when the shutter is closed due to its high vapor pressure or can evaporate from regions surrounding the effusion cell that are being warmed up. Once the temperatures of the effusion cells have reached the operating temperature, the Cs shutter is opened. Upon exposure to the direct Cs flux, the photocurrent from GaAs suddenly increases, reaching a peak abruptly, and then decreasing slowly. At this point, pure oxygen is leaked into the chamber with slowly increasing relative pressure to maximize the growth of the photocurrent signal, as is typically performed in $\mathrm{Cs}-\mathrm{O}_{2}$ co-deposition activation of GaAs [22]. In our experimental conditions, the $\mathrm{O}_{2}$ partial pressure has to be raised to about $5 \times 10^{-9}$ Torr. Upon simultaneous exposure to Cs and oxygen flux, the quantum efficiency rises again until a maximum value is reached. Then, the Sb shutter is also opened for a well defined time that corresponds to a dose that we quantify in terms of equivalent Sb thickness. Based on the calibration of our effusion cell, the quantities of $\mathrm{Sb}$ deposited on the GaAs substrates surface were equivalent to $0.12,0.25,0.37$ and $0.50 \mathrm{~nm}$ thicknesses. During $\mathrm{Sb}$ evaporation, the photocurrent decreases. After the Sb shutter is closed, the photocurrent increases again until a plateau is reached. At this point, the heater is turned off to initiate the cooling of the photocathode and favor further intake of Cs. The extracted photocurrent is seen to increase again until a second plateau is reached. Then, also the oxygen valve is closed and only Cs flux is left directed the photocathode surface. The Cs shutter is closed once the temperature has lowered enough that the photocurrent is stable.

Once the growth of the activating layer is complete, the samples are moved under vacuum into the Auger/LEED vacuum chamber (see Fig. 2) with a base pressure of $5 \times 10^{-11}$ Torr. Here the samples are held at a negative bias and illuminated by light generated with a lamp and monochromator to measure the quantum efficiency as a

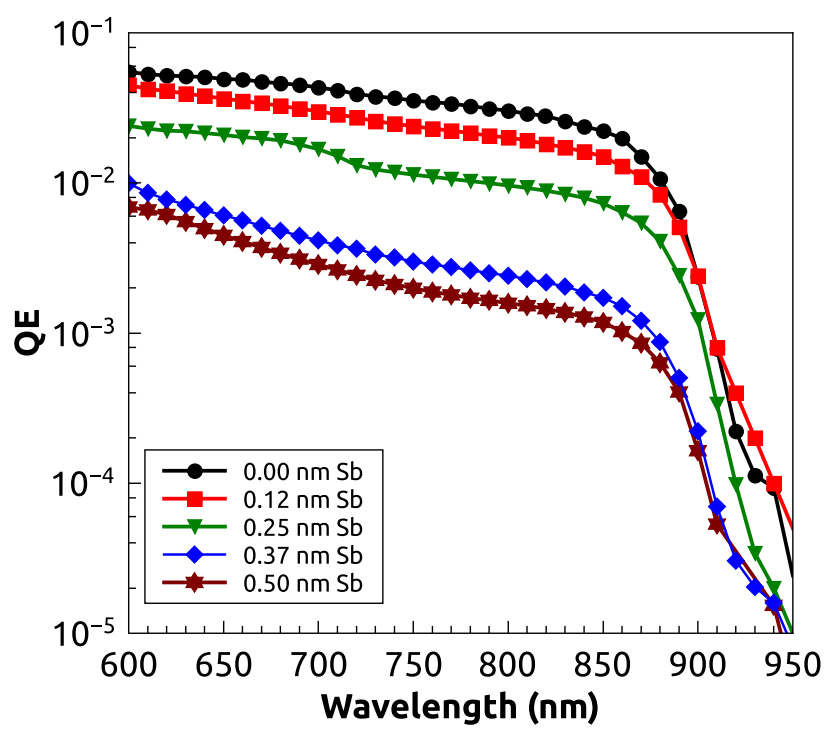

FIG. 4. Spectral response of the 5 samples as measured after growth. While NEA is present in all the samples, demonstrated by sensitivities extending well beyond the $875 \mathrm{~nm}$ (corresponding to the nominal value of the GaAs band gap of $1.42 \mathrm{eV}$ ), the overall efficiency decreases as the equivalent thickness of the Sb layer increases.

function of wavelength. The spectral responses of the samples measured after the growth are reported in Fig. 4. Between different samples the QE decreases by roughly one order of magnitude as the $\mathrm{Sb}$ dose was increased during the growth. Nevertheless, NEA conditions are still preserved in all samples, as shown by significant QE extending beyond wavelengths longer than $875 \mathrm{~nm}$, which corresponds to the nominal band gap value of $1.42 \mathrm{eV}$ for GaAs. The observed decrease of the QE as the $\mathrm{Sb}$ thickness is increased may indicate the presence of a relaxation mechanism in the activating layer that filters out an increasing number of electrons as the thickness grows.

After the spectral response was measured, every sample was moved under vacuum into the vacuum chamber which houses a Mott polarimeter (see Fig. 2) [23]. This Mott analyzers Sherman function was characterized to be $\mathrm{S}_{\text {eff }}=$ 0.15 at $20 \mathrm{kV}$ operating voltage with a $5 \%$ accuracy when electrons within a $1 \mathrm{keV}$ energy loss window are detected [24]. Monochromatic light was circularly polarized using a linear polarizer (Thorlabs LPVIS050) and a liquid crystal variable wave plate (Thorlabs LC1113B). It illuminates the sample surface at normal incidence. The helicity of circular polarization was switched by alternating the retardance of the liquid crystal wave plate between $1 / 4 \lambda$ and $3 / 4 \lambda$.

The electron spin polarization (ESP) as a function of illumination wavelength for each sample are reported in Fig. 5.

These ESP results confirm that the NEA of the samples is preserved even with an increased overall thickness of the activating layer. This is consistent with the shape of 


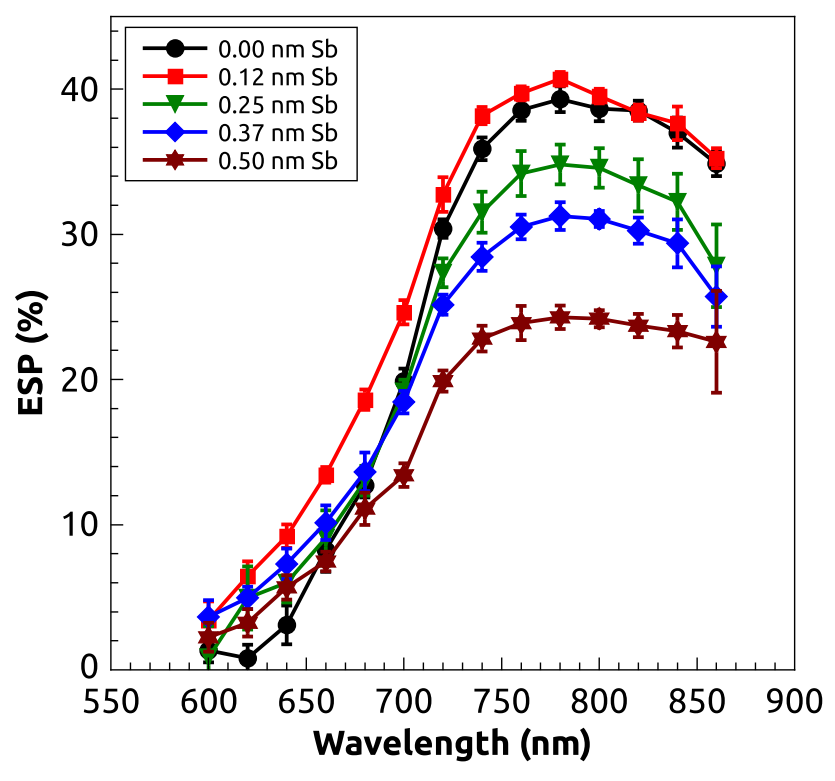

FIG. 5. Measured electron spin polarization for the five samples as a function of the illuminating wavelength. The maximum achievable spin polarization (measured at about $780 \mathrm{~nm}$ ) decreases as the equivalent thickness of the Sb increases beyond $0.12 \mathrm{~nm}$.

all of the ESP curves which show a characteristic maximum around $780 \mathrm{~nm}$. A decrease of the peak value of the spin polarization is observed as the activating layer grows thicker.

Finally, the robustness of the Sb-Cs-O activated samples has been evaluated by performing QE lifetime measurements (see Fig. 6). These lifetimes are then compared to the lifetime of a Cs-O activated GaAs. The light of a $505 \mathrm{~nm}$ laser was sent to the surface of all the photocathodes held at the negative bias of $-18 \mathrm{~V}$ in the Auger/LEED vacuum chamber with a base pressure of $5 \times 10^{-11}$ Torr. The laser power was adjusted to initially extract photocurrents in the range between 200 and $300 \mathrm{nA}$. Photocurrent was measured until it decreased more than a factor of 3 (as it was the case of the Cs-O activated GaAs) or until the extracted charge becomes larger than $0.02 \mathrm{C}$ (as it was the case for all the Sb-Cs-O activated GaAs samples). QE measurements were conducted also with a $2 \%$ duty cycle over extended period of time to estimate the dark lifetime of the samples in $5 \times 10^{-11}$ Torr vacuum. Using these two QE measurements, we were able to measure the robustness of the SbCs-O activating layer with respect to both the chemical poisoning via the dark life time, and the ion back bombardment via the charge extraction lifetime. In a simple model to account for the QE decay, we assume that efficiency will decrease exponentially as a function of time because of 2 factors. First, the continuous exposure to residual gasses in the UHV chamber eventually leads to chemical poisoning of the photocathode surface. Second, the beam can ionize the residual gas molecules and produces positively charged species which are accelerated toward the cathode and strike

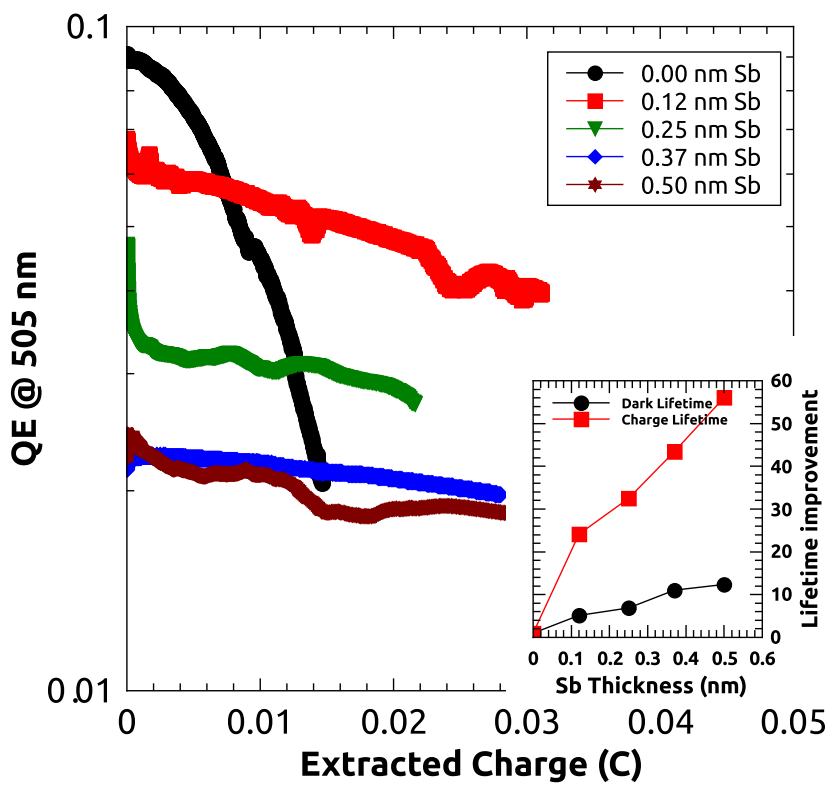

FIG. 6. QE as a function of the extracted charge as measured for the different samples at $505 \mathrm{~nm}$. The data shows that as the thickness of the activating layer is increased, the initial QE value decreases but the lifetime is increased. The inset shows a plot of the lifetime improvement achieved with respect to the Cs-O activated sample for dark lifetime $\left(\tau_{D L}\right)$ and charge lifetime $\left(\tau_{Q}\right)$.

its surface. This second mechanism is a function of the total charge removed from the photocathode. With this, we write the QE as a function of time ( $\mathrm{t}$ ) and extracted charge $(\mathrm{Q})$ as

$$
Q E(t, Q)=Q E_{i} \times e^{-t / \tau_{D L}} \times e^{-Q / \tau_{Q}}
$$

where $\mathrm{QE}_{i}, \tau_{D L}, \tau_{Q}$ are the initial $\mathrm{QE}$, the dark lifetime, and the charge extraction lifetime respectively. Estimates of the $\tau_{D L}$ values are obtained by measuring the $\mathrm{QE}$ of the samples with a low duty cycle. The $\tau_{D L}$ values obtained by exponential fit of the experimental data are then used in the exponential fit of the QE data obtained with a $100 \%$ duty cycle to estimate the charge extraction lifetime $\tau_{Q}$.

\section{DISCUSSION}

Our measurements show that this method of activation yields a more robust activation layer, which is in line with previous findings [13]. In addition, we have characterized the increased lifetime by estimating both $\tau_{D L}$ and $\tau_{Q}$ as Sb thickness is increased as reported in Table I. While the full lifetime characterization was performed at $505 \mathrm{~nm}$, spectral response measurements performed before and after the extended lifetime measurements indicate that decay constants similar to the ones measured at $505 \mathrm{~nm}$ also capture the rate of QE decrease at photon energies in the IR, which are relevant for the production of spin polarized electrons. Figure 7 reports the QEs measured at $780 \mathrm{~nm}$ before and after the lifetime measurements that are reported in Fig. 6. While we have not estimated the dark and charge lifetime 
TABLE I. Values for $\tau_{D L}$ and $\tau_{Q}$ and relative gains in lifetime with respect to the $\mathrm{Cs}-\mathrm{O}$ activated sample as obtained from lifetime measurements performed using a $505 \mathrm{~nm}$ laser diode.

\begin{tabular}{lcccc}
\hline \hline Sb thickness $(\mathrm{nm})$ & $\tau_{D L}$ (hour) & $\tau_{Q}$ (Coulomb) & Gain $_{D L}$ & Gain $_{Q}$ \\
\hline 0.00 & 29 & 0.01 & 1 & 1 \\
0.12 & 147 & 0.25 & 5 & 25 \\
0.25 & 197 & 0.33 & 6.8 & 33 \\
0.37 & 316 & 0.45 & 10.9 & 45 \\
0.50 & 354 & 0.58 & 12.2 & 58 \\
\hline \hline
\end{tabular}

separately at $780 \mathrm{~nm}$, data in Fig. 7 show that similar trends are observed at both wavelengths.

We can define a figure of merit (FOM) for GaAs photocathodes for spin polarized electron beam production to include a factor that takes into account the lifetime gain induced by the activating layer [6]:

$$
\mathrm{FOM}=\mathrm{QE} \times \mathrm{ESP}^{2} \times \tau
$$

The choice of which $\tau$ should relate to the mechanism that likely will limit lifetime in the operating conditions. For low current applications it is likely that lifetime will be dominated by chemical poisoning while in high average current operation ion back bombardment will be the limiting factor. Using this formula with either $\tau_{D L}$ or $\tau_{Q}$ lifetimes as measured in similar operational conditions

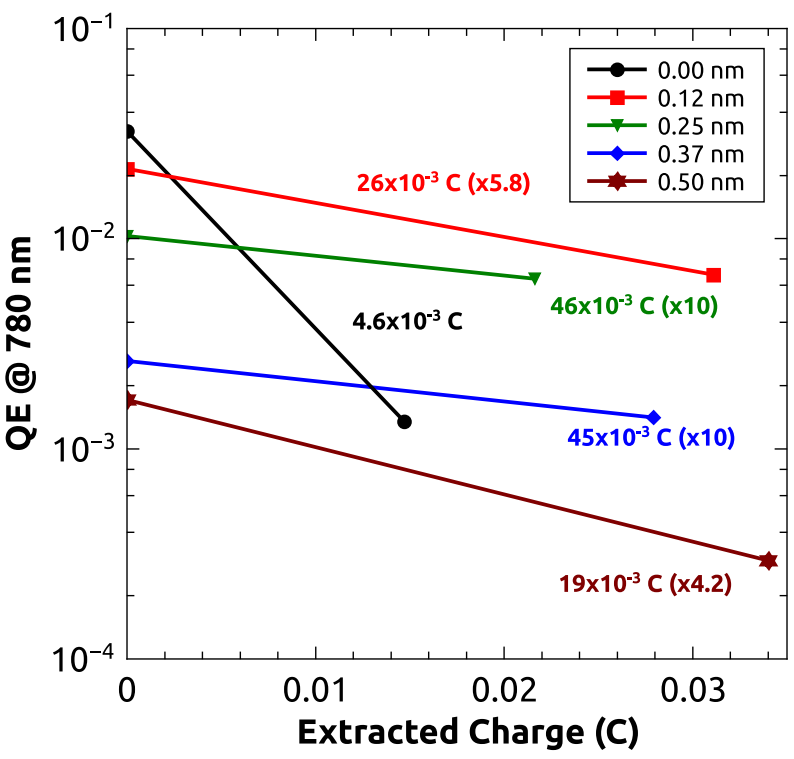

FIG. 7. QEs measured at $780 \mathrm{~nm}$ before and after the lifetime measurements that are reported in Fig. 6. From the QE measurements at $780 \mathrm{~nm}$ we can estimate the lifetimes as function of the extracted charge and relative gains with respect to the Cs-o activates sample. These values are provided alongside the data. Estimated lifetime gains are similar to the ones observed at $505 \mathrm{~nm}$. yields a classification of the performance of different photocathodes for a specific application. In our experimental conditions and in the range of beam currents used, the FOMs of the photocathode prepared using $0.12 \mathrm{~nm}$ of $\mathrm{Sb}$, are respectively larger by a factor of 4 and by a factor of 16 with respect to the standard GaAs activation.

Direct measurements in a real photogun environment should be performed to confirm the robustness improvements of the $\mathrm{Cs}_{3} \mathrm{Sb}$ activated samples beyond the standard activation. Nevertheless, factors like the electron beam energy and source vacuum pressure, that limit the photocathode lifetimes measured in our experiment, can be accounted for allowing extrapolating an estimate of the expected lifetimes in a real electron gun. Other factors, like the effects of damage induced by the back streaming ions in the case of the much higher energy are more challenging to accurately estimate.

At the electron energy we produced during our experiment, the electron-impact ionization cross section is near its maximum and it is about 3 orders of magnitude larger than that of beams produced by DC gun operating at few hundreds of $\mathrm{kV}$ [25-27]. Also, in a typical electron gun for polarized electron beams, the static vacuum level is more than 50 times lower than the one used in our experiment [25]. The dark lifetimes of the photocathodes used in the experiment here which depend only on the static vacuum pressure should scale approximately as the inverse of the value achieved in a real photogun. This yields expected dark lifetimes of at least 600 hours and 3000 hours for the GaAs activated with only Cs-O and with the $\mathrm{Cs}_{3} \mathrm{Sb}$ layer with $0.12 \mathrm{~nm}$ of $\mathrm{Sb}$ respectively.

If we assume that the measured charge lifetimes are inversely proportional to the number of ions produced by electron-impact ionization and we use typical vacuum pressures and beam energies of modern high voltage DC guns to calculate the number of ions, we find that the charge lifetimes will be larger by about 4 orders of magnitude with respect to the value we measured in our vacuum system. This yields an expected charge lifetime of $100 \mathrm{C}$ and $2.5 \mathrm{kC}$ for $\mathrm{Cs}-\mathrm{O}$ and $\mathrm{Cs}_{3} \mathrm{Sb}$ layer with $0.12 \mathrm{~nm}$ of $\mathrm{Sb}$ respectively. This simple scaling of the lifetimes for Cs-O yield numbers that are within the range of values previously reported in literature [25-28].

The improved robustness of the activating layer based on Sb-Cs-O could be related to the fact that the NEA is achieved by a proper alignment of the bands in a heterojunction between the two semiconductors with an activating layer that has a much larger thickness than the typical Cs-O layer. The observed improved resistance to the ion back bombardment may be due to the increased thickness of the activating layer in a similar fashion to the alkali antimonide photocathodes [29]. The improved resilience to chemical poisoning which yields larger dark lifetimes could be due to the fact that the surface of alkali antimonides is less chemically reactive than the $\mathrm{Cs}-\mathrm{O}$ layer. 
The thickest layer we have grown on GaAs is estimated to be approximately $3 \mathrm{~nm}$ based on the equivalent $\mathrm{Sb}$ thickness deposited and the value of the expansion coefficient for $\mathrm{Cs}_{3} \mathrm{Sb}$ formation, which is reported to be about 6 [30]. Electrons injected in to $\mathrm{Cs}_{3} \mathrm{Sb}$ from GaAs should have kinetic energies larger than the electron affinity of the activating layer to be emitted into vacuum. Let us assume this energy to be equal to $0.45 \mathrm{eV}$ as in the case of $\mathrm{Cs}_{3} \mathrm{Sb}$, though it should be noted that it is possible that surface oxidation resulting from our preparation procedure can lower this value slightly [31]. Hot carriers that have been injected from GaAs across the heterojunction barrier have transition times in the $\mathrm{Cs}_{3} \mathrm{Sb}$ on the order of a few tens of femtoseconds. On such short timescales, electron spin depolarization occurring through the D'yakonov-Perel and Bir-Aronov-Pikus mechanisms is negligible [32].

While more detailed characterizations of the activation layer are planned to confirm our hypotheses, one of the possible explanations for the simultaneous decrease of $\mathrm{QE}$ and spin polarization observed as the thickness of the activation layer is increased may involve intercarrier energy exchange between two electron populations out of equilibrium: the population of hot electrons injected from GaAs and the one of photoexcited electrons in the conduction band of the activation layer [33,34]. Unlike our previous experiments where we used a much larger energy gap material like $\mathrm{Cs}_{2} \mathrm{Te}$ [15] to form a NEA surface in GaAs with a similar layer thickness and for which no electron spin depolarization has been observed, in the $\mathrm{Cs}_{3} \mathrm{Sb}$ case electrons can be excited to the conduction band at photon energies slightly larger than $1.6 \mathrm{eV}$ due to the presence of intra-gap impurity states [18].

The extinction coefficient for GaAs at $780 \mathrm{~nm}$ is about $2 \times 10^{4} \mathrm{~cm}^{-1}$ [35], one order of magnitude lower than the one reported for $\mathrm{Cs}_{3} \mathrm{Sb}$, which depends on experimental growth conditions and ranges from 1 to $5 \times 10^{5} \mathrm{~cm}^{-1}$ [19]. Our polarization measurements were conducted in continuous wave photoemission conditions using the light produced from a discharge lamp and a monochromator. At $780 \mathrm{~nm}$, we typically illuminate the sample with about 100 $\mathrm{nW}$ and $3 \mathrm{~mm}$ diameter spot size and extract about $1 \mathrm{nA}$ of photocurrent from the samples. Assuming an absorption depth of $1 \mu \mathrm{m}$, the average density of photoexcited electron in GaAs is about $3 \times 10^{17} \mathrm{~cm}^{-3}$. Assuming $3 \%$ transmission across the heterojunction interface (which is consistent with the QE of GaAs activated with Cs-O only), the steady state density of hot electrons injected at the interface should be roughly $1 \times 10^{16} \mathrm{~cm}^{-3}$. Additionally, part of the incident light at $780 \mathrm{~nm}$ is absorbed by the few $\mathrm{nm}$ of $\mathrm{Cs}_{3} \mathrm{Sb}$. Using the same reasoning, but now assuming one order of magnitude larger absorption coefficient (i.e $2 \times 10^{5} \mathrm{~cm}^{-1}$ ) in the case of the sample with $0.12 \mathrm{~nm}$ of $\mathrm{Sb}$ equivalent thickness, $2 \%$ of the light is absorbed by the $\mathrm{Cs}_{3} \mathrm{Sb}$. Given the small thickness of this layer, the density of photoexcited electrons resulting from this absorption may reach values of $1 \times 10^{19} \mathrm{~cm}^{-3}$ in $\mathrm{Cs}_{3} \mathrm{Sb}$. In terms of kinetic energy, electrons belonging to this second population are already almost fully relaxed to the bottom of the conduction band.

Based on the diagram in Fig. 8, we suspect that under our experimental conditions, electrons that are extracted into vacuum are either, (1) ballistic and transported across the activating layer without energy losses, or (2) are quasiballistic and move across the activation layer where they suffer one or more scattering events with the lattice or other electrons or else (3) are coming from an energy exchange scattering process with a low energy electron which has been photoexcited in the conduction band of the activating layer. The ballistic electrons will preserve the spin polarization achieved in GaAs while the quasi-ballistic electrons and electrons coming from an exchange energy process can contribute to the measured depolarization. Using Poisson statistics, during a time duration $\Delta t$, the probability of electrons traveling without a single scattering is $e^{-\Gamma_{e e} \Delta t}$ where $\Gamma_{e e}$ is the electron-electron scattering rate. Then, the probability of electrons experiencing scattering with another electron at least once would be $1-e^{-\Gamma_{e e} \Delta t}$. If we assume that hot electrons which participate in one or more scattering event in the activation layer can lead to spin relaxation because they lose their original spin information, the expected value of spin polarization $\Delta \mathrm{ESP}_{e e}$ change due to electron scattering in a time interval $\Delta t$ can be written as

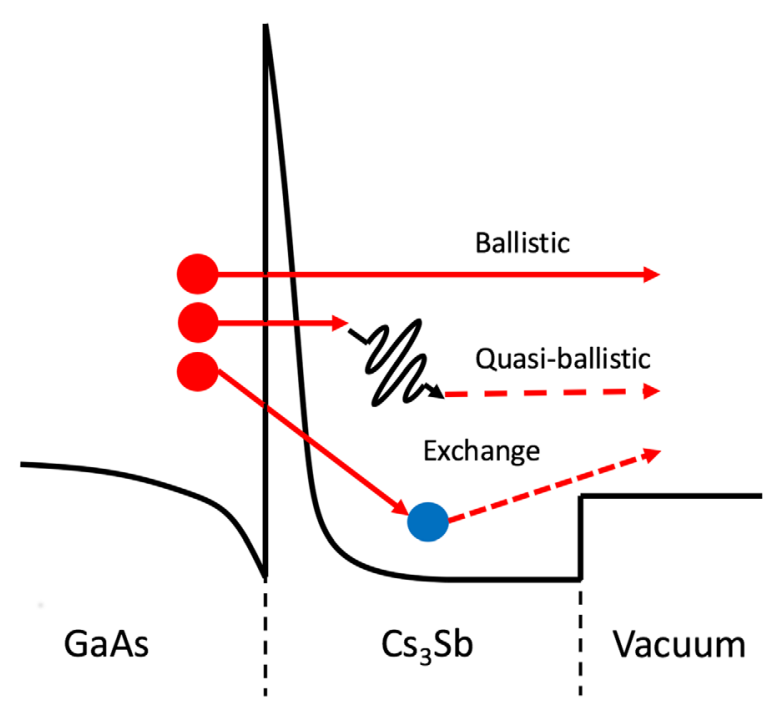

FIG. 8. Schematic representation of the three different processes leading to the extraction of photoelectrons from GaAs, through the $\mathrm{Cs}_{3} \mathrm{Sb}$ layer into vacuum. Ballistic hot electrons travel trough the activation layer without interaction with lattice or electrons; quasi-ballistic electrons can be still extracted if few scattering events take place so that they have sufficient energy to overcome the barrier to vacuum; hot electrons can exchange energy with low energy electrons and give them sufficient energy to escape into vacuum. 


$$
\Delta \mathrm{ESP}_{e e}=-\operatorname{ESP}(0)\left(1-e^{-\Gamma_{e e} \Delta t}\right) .
$$

Thus,

$$
\operatorname{ESP}(t) \approx \operatorname{ESP}(0)+\Delta \operatorname{ESP}_{e e}=\operatorname{ESP}(0) e^{-\Gamma_{e e} t} .
$$

If we assume that the transit time of electrons which tunnel from GaAs into the activating layer is given by their kinetic energy $(E)$ and the expected thickness of the activating layer, then:

$$
\operatorname{ESP}(L, E)=\operatorname{ESP}(0) e^{-\Gamma_{e e} L / \sqrt{2 E / m}}
$$

where $\operatorname{ESP}(0)$ is the electron spin polarization measured in the case activation with a Cs-O layer that we assume having negligible thickness. As the thickness of the activation layer is increased, the probability of ballistic electron transmission decreases while the probability for electrons to suffer one or more scattering events and hence experience depolarization increases.

Figure 9 reports a comparison of our experimental data with the analytical prediction obtained from Eq. (5). Given the simplified description, the agreement is not perfect, but, Eq. (5) is capable of capturing most of the features characterizing our experimental ESP data. The value of $\Gamma_{e e}$ is assumed to be constant and equal to $1.5 \times 10^{14} \mathrm{~s}^{-1}$ and is consistent with experimental measurements [36] indicating that photogenerated hot electrons in metals can thermalize with characteristic times even shorter than 10 fs through carrier-to-carrier or carrier-to-phonon scattering. Similar relaxation times have also been measured in the case of lead-iodide hybrid perovskite, a semiconductor having

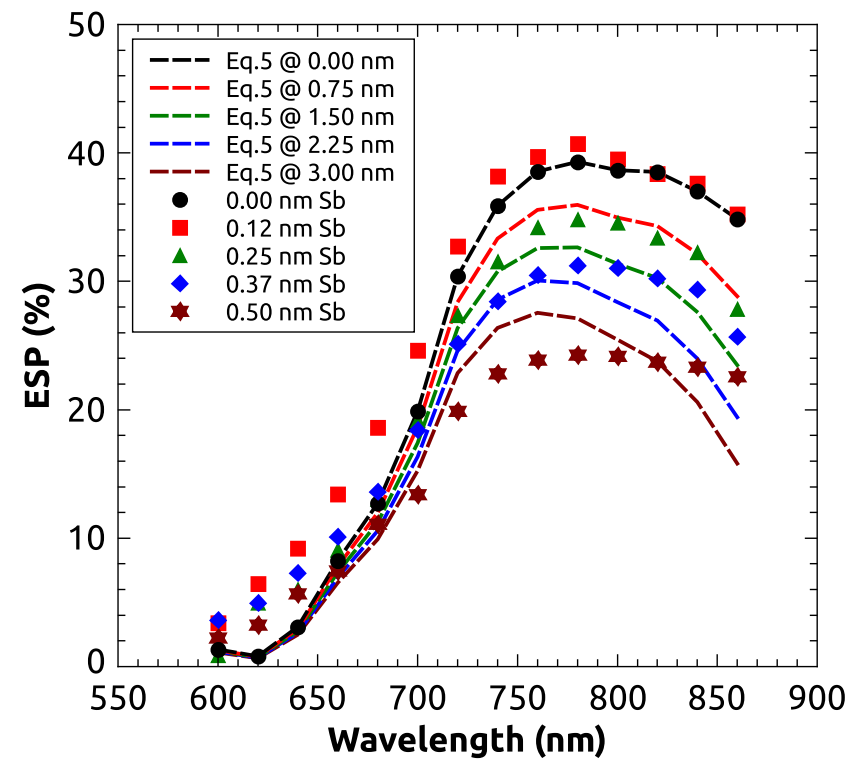

FIG. 9. ESP of the samples activated to NEA with $\mathrm{Cs}_{3} \mathrm{Sb}$ estimated using Eq. (5). The used value of $\Gamma_{e e}$ is reported in Table II. similar band gap energy to $\mathrm{Cs}_{3} \mathrm{Sb}$ for hot electrons with excess energies on the order of $0.5 \mathrm{eV}$ [37].

The probability of tunneling across a triangular shaped barrier can be estimated using the Wentzel-KramersBrillouin (WKB) approximation. In the case of a triangular barrier with a height $\Phi$ and width $w$ the tunneling probability across such barrier $T(E)$ of one electron with kinetic energy $E$ can be written as:

$$
T(E)=T_{0} e^{E / E_{0}}
$$

here

$$
T_{0}=e^{-\frac{4 w}{3}} \sqrt{\frac{2 m \Phi}{\hbar^{2}}}
$$

and

$$
E_{0}=\frac{1}{2 w} \sqrt{\frac{\hbar^{2} \Phi}{2 m}}
$$

For a width of $0.15 \mathrm{~nm}$ and a value of $\Phi$ equal to the electron affinity of GaAs $(4.07 \mathrm{eV})$, Eq. (6) yields a tunneling probability that is consistent with the measured QE of GaAs activated with Cs-O. Larger values for the width of the barrier are required to reproduce QEs from NEA activating $\mathrm{Cs}_{3} \mathrm{Sb}$ layers [20].

If we assume that scattering losses inside the activating layer are negligible, then the quantum efficiency $Q E\left(w_{\mathrm{Cs}_{3} \mathrm{Sb}}\right)$ relative to a barrier width $w_{\mathrm{Cs}_{3} \mathrm{Sb}}$ corresponding to a thickness of the activating layer $L_{\mathrm{Cs}_{3} \mathrm{Sb}}$ can be modeled as:

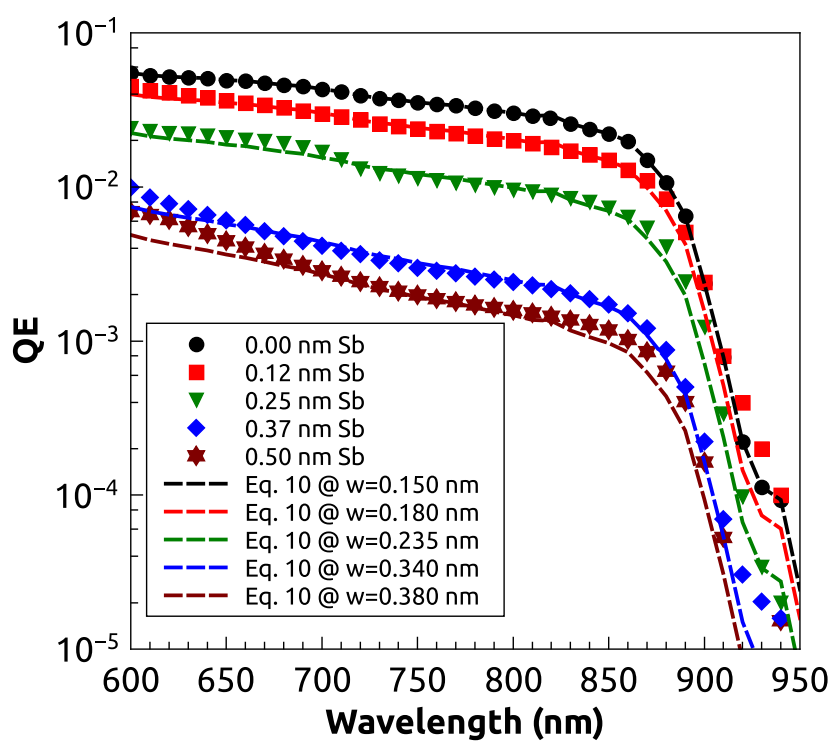

FIG. 10. Spectral response of the samples activated to NEA with $\mathrm{Cs}_{3} \mathrm{Sb}$ and the corresponding prediction of Eq. (10). Barrier thicknesses are given in Table II. 
TABLE II. Parameters values used to model QE and ESP using Eq. (5) and (10).

\begin{tabular}{lc}
\hline \hline Parameter & Value \\
\hline$\Phi(\mathrm{eV})$ & 4.07 \\
$w_{0}(\mathrm{~nm})$ & 0.150 \\
$w_{0.75}(\mathrm{~nm})$ & 0.180 \\
$w_{1.50}(\mathrm{~nm})$ & 0.235 \\
$w_{2.25}(\mathrm{~nm})$ & 0.340 \\
$w_{3.00}(\mathrm{~nm})$ & 0.380 \\
$\Gamma_{e e}\left(\mathrm{~s}^{-1}\right)$ & $1.5 \times 10^{14}$ \\
\hline \hline
\end{tabular}

$$
Q E\left(w_{\mathrm{Cs}_{3} \mathrm{Sb}}\right)=Q E_{0} \frac{T\left(w_{\mathrm{Cs}_{3} \mathrm{Sb}}\right)}{T\left(w_{0}\right)}
$$

where $Q E_{0}$ is the $\mathrm{QE}$ measured for the Cs-O activated sample, $T\left(w_{\mathrm{Cs}_{3} \mathrm{Sb}}\right)$ and $T\left(w_{0}\right)$ are the WKB tunneling probability for the triangular barrier of width $w_{\mathrm{Cs}_{3} \mathrm{Sb}}$ in the case of $\mathrm{Cs}_{3} \mathrm{Sb}$ with a thickness $L_{\mathrm{Cs}_{3} \mathrm{Sb}}$ and of width $w_{0}$ in the case $\mathrm{Cs}-\mathrm{O}$ activating layer.

Then, Eq. (9) can be rewritten as

$$
Q E\left(E, w_{\mathrm{Cs}_{3} \mathrm{Sb}}\right)=Q E_{0}(E) \frac{T_{0}\left(w_{\mathrm{Cs}_{3} \mathrm{Sb}}\right) e^{\frac{E}{\bar{E}_{0}\left(w_{\mathrm{C}_{3} \mathrm{Sb}}\right)}}}{T_{0}\left(w_{0}\right) e^{\frac{E}{E_{0}\left(w_{0}\right)}}}
$$

to explicitly include also the dependence on the initial energy $\mathrm{E}$ of photoelectrons in the GaAs.

Using the spectral response obtained from GaAs activated with Cs-O and a fixed value for the barrier width $w_{0}$

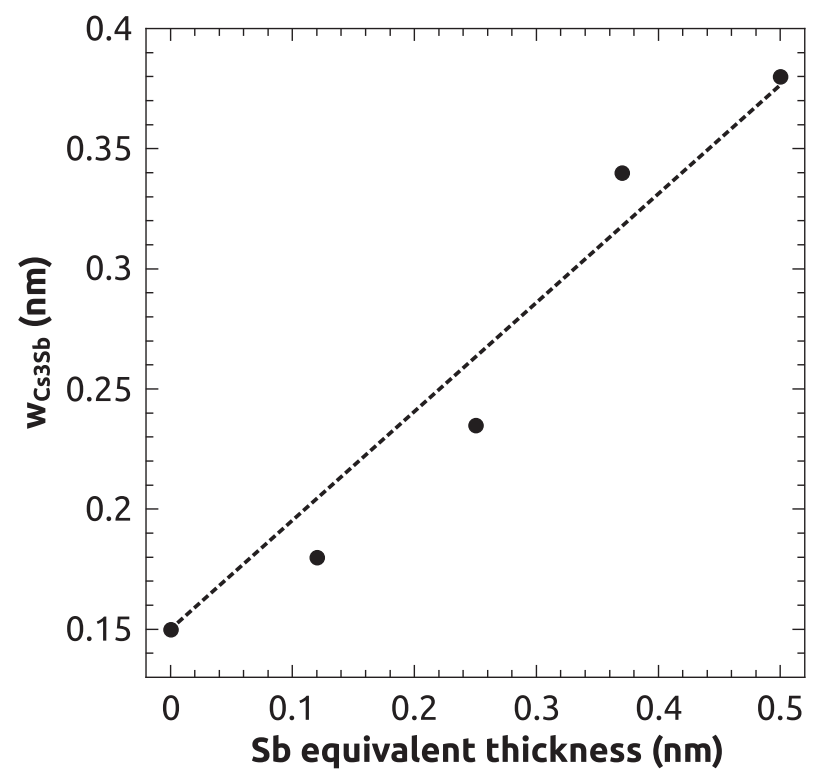

FIG. 11. The fit values of $w_{\mathrm{Cs}_{3} \mathrm{Sb}}$ used in Fig. 10, plotted as a function of the equivalent thickness of the Sb layer. A roughly linear trend is noted, supporting the conclusion that a thicker activating layer weakens the electric dipole at the interface between $\mathrm{GaAs}$ and $\mathrm{Cs}_{3} \mathrm{Sb}$. value for GaAs (that we assume to be $0.15 \mathrm{~nm}$ [20]), we can use Eq. (10) to fit the width of the barriers corresponding to the different thicknesses of the $\mathrm{Cs}_{3} \mathrm{Sb}$ activating layer that allows to best reproduce the experimental data.

Fit values of the widths are shown in Table II. We find that Eq. (10) gives good agreement with the measured QEs at each width. Figure 11 plots the fit barrier width values as a function of the equivalent thickness of $\mathrm{Sb}$. We note a roughly linear trend. This may suggest that the segregation of the initial layer of Cs-O becomes less sharp as the $\mathrm{Cs}_{3} \mathrm{Sb}$ layer grows thicker.

While conceptually simple, the models of ESP and QE given Eq. (5) and Eq. (10) give surprisingly good agreement with our experimental data. A more accurate description would require more advanced numerical modeling of electron transport, which is outside the scope of this work.

\section{OUTLOOK AND CONCLUSIONS}

In this work, we report that GaAs activated to NEA with $\mathrm{Cs}, \mathrm{Sb}$ and $\mathrm{O}$ yields longer lifetimes than the traditional Cs-O activation under similar experimental conditions. We measure and discuss trade-offs of quantum efficiency and spin polarization as function of the activation layer thickness.

Further studies involving numerical simulations of electron transport and surface characterization are planned. Additionally, we plan to fully characterize these heterojunctions in the operating conditions of a real photoinjector to confirm the measured lifetime increases. Finally, we intend to explore the applicability of this activation method to SL-based GaAs photocathodes. The possibility to create photocathodes that can provide one order of magnitude longer operational lifetimes opens exiting new avenues for the production and application of intense highly polarized electron beams.

\section{ACKNOWLEDGMENTS}

This work was supported by DOE under Award No. DESC0019122 and by NSF under Grant No. PHY-1549132.

[1] Report of the community review of eic accelerator $r \& d$ for the office of nuclear physics, https://doi.org/10.2172/ 1367855 (2017).

[2] Reaching for the horizon: The 2015 long range plan for nuclear science, https://www.osti.gov/biblio/1296778reaching-horizon-long-range-plan-nuclear-science (2015).

[3] Major nuclear physics facilities for the next decade: Report of the nsac subcommittee on scientific facilities, https:// science.osti.gov/-/media/np/nsac/pdf/docs/2013/NSAC_ FacilitiesReport.pdf (2013).

[4] M. Kuwahara, S. Kusunoki, Y. Nambo, K. Saitoh, X. Jin, T. Ujihara, H. Asano, Y. Takeda, and N. Tanaka, Coherence of a spin-polarized electron beam emitted from 
a semiconductor photocathode in a transmission electron microscope, Appl. Phys. Lett. 105, 193101 (2014).

[5] N. Rougemaille and A. Schmid, Magnetic imaging with spin-polarized low-energy electron microscopy, Eur. Phys. J. Appl. Phys. 50, 20101 (2010).

[6] W. Liu, Y. Chen, W. Lu, A. Moy, M. Poelker, M. Stutzman, and S. Zhang, Record-level quantum efficiency from a high polarization strained GaAs/GaAsP superlattice photocathode with distributed Bragg reflector, Appl. Phys. Lett. 109, 252104 (2016).

[7] D. T. Pierce, F. Meier, and P. Zrcher, Negative electron affinity GaAs: A new source of spin-polarized electrons, Appl. Phys. Lett. 26, 670 (1975).

[8] Z. Liu, Y. Sun, S. Peterson, and P. Pianetta, Photoemission study of $C s-N F_{3}$ activated GaAs(100) negative electron affinity photocathodes, Appl. Phys. Lett. 92, 241107 (2008).

[9] L. S. Cardman, The PEPPo method for polarized positrons and PEPPo II, AIP Conf. Proc. 1970, 050001 (2018).

[10] I. V. Bazarov, B. M. Dunham, Y. Li, X. Liu, D. G. Ouzounov, C. K. Sinclair, F. Hannon, and T. Miyajima, Thermal emittance and response time measurements of negative electron affinity photocathodes, J. Appl. Phys. 103, 054901 (2008).

[11] L. Zhao, Q. Zhou, S. Zhao, and B. Xie, Proceedings SPIE 1982, Photoelectronic Detection and Imaging: Technology and Applications '93 1982 (1993), p. 140, https://doi.org/ 10.1117/12.142006.

[12] M. Hagino and R. Nishida, Photoemission from GaAs-CsSb (Te), Jpn. J. Appl. Phys. 8, 123 (1969).

[13] T. Guo, Novel GaAs photocathodes with alkali antimonide intermediate layers and caesium-oxygen adlayers, Thin Solid Films 281-282, 379 (1996).

[14] H. Sonnenberg, On Photoemission from GaAs-Cs-Sb, Jpn. J. Appl. Phys. 8, 806 (1969).

[15] J. K. Bae, L. Cultrera, P. DiGiacomo, and I. Bazarov, Rugged spin-polarized electron sources based on negative electron affinity GaAs photocathode with robust $\mathrm{Cs}_{2} \mathrm{Te}$ coating, Appl. Phys. Lett. 112, 154101 (2018).

[16] M. Kuriki and K. Masaki, Negative electron affinity GaAs cathode activation with CsKTe thin film, Proceedings of 10th International Particle Accelerator Conference (JACoW Publishing, Geneva, Switzerland, 2019), p. 1986, https://doi.org/10.18429/JACoW-IPAC2019-TUPTS026.

[17] O. Rahman, M. Gaowei, W. Liu, E. Wang, and J. Biswas, New activation techniques for higher charge lifetime from GaAs photocathodes, Proceedings of 10th International Particle Accelerator Conference (2019), p. 2157, https:// doi.org/10.18429/JACoW-IPAC2019-TUPTS102.

[18] W. E. Spicer, Photoemission and Related Properties of the Alkali-Antimonides, J. Appl. Phys. 31, 2077 (1960).

[19] W. E. Spicer, Photoemissive, Photoconductive, and Optical Absorption Studies of Alkali-Antimony Compounds, Phys. Rev. 112, 114 (1958).

[20] J. K. Bae, A. Galdi, L. Cultrera, F. Ikponmwen, J. Maxson, and I. Bazarov, Improved lifetime of a high spin polarization superlattice photocathode, arXiv:1911.09609.

[21] M. Rei Vilar, J. El Beghdadi, F. Debontridder, R. Artzi, R. Naaman, A. M. Ferraria, and A. M. B. Do Rego,
Characterization of wet-etched GaAs (100) surfaces, Surf. Interface Anal. 37, 673 (2005).

[22] F. Machuca, Z. Liu, Y. Sun, P. Pianetta, W. E. Spicer, and R. F. W. Pease, Role of oxygen in semiconductor negative electron affinity photocathodes, J. Vac. Sci. Technol. B 20, 2721 (2002).

[23] T. Maruyama, A. Brachmann, J. Clendenin, T. Desikan, E. Garwin, R. Kirby, D.-A. Luh, J. Turner, and R. Prepost, A very high charge, high polarization gradient-doped strained GaAs photocathode, Nucl. Instrum. Methods Phys. Res., Sect A 492, 199 (2002).

[24] G. Mulhollan, Proceedings of the workshop on photocathodes for polarized electron sources and accelerators (1994), p. 211, http://inspirehep.net/record/373713/files/ slac-r-432.pdf.

[25] C. K. Sinclair, P. A. Adderley, B. M. Dunham, J. C. Hansknecht, P. Hartmann, M. Poelker, J. S. Price, P. M. Rutt, W. J. Schneider, and M. Steigerwald, Development of a high average current polarized electron source with long cathode operational lifetime, Phys. Rev. Accel. Beams 10, 023501 (2007).

[26] R. Alley et al., The Stanford linear accelerator polarized electron source, Nucl. Instrum. Methods Phys. Res., Sect. A 365, 1 (1995).

[27] K. Aulenbacher et al., The MAMI source of polarized electrons, Nucl. Instrum. Methods Phys. Res., Sect. A 391, 498 (1997).

[28] R. Suleiman, P. Adderley, J. Grames, J. Hansknecht, M. Poelker, and M. Stutzman, High current polarized electron source, AIP Conf. Proc. 1970, 050007 (2018).

[29] M. A. A. Mamun, A. A. Elmustafa, C. Hernandez-Garcia, R. Mammei, and M. Poelker, Effect of Sb thickness on the performance of bialkali-antimonide photocathodes, J. Vac. Sci. Tech. A 34, 021509 (2016).

[30] M. Hagino and T. Takahashi, Thickness of Cs-Sb Films Relative to the Original Sb Films, J. Appl. Physics 37, 3741 (1966).

[31] A. Sommer, Photo-electric alloys of alkali metals, Proc. Phys. Soc. 55, 145 (1943).

[32] W. Liu, M. Poelker, X. Peng, S. Zhang, and M. Stutzman, A comprehensive evaluation of factors that influence the spin polarization of electrons emitted from bulk GaAs photocathodes, J. Appl. Phys. 122, 035703 (2017).

[33] C. J. Hearn, Inter-carrier energy exchange and the critical concentration of hot carriers in a semiconductor, Proc. Phys. Soc. 86, 881 (1965).

[34] A. F. J. Levi, J. R. Hayes, P. M. Platzman, and W. Wiegmann, Injected-Hot-Electron Transport in GaAs, Phys. Rev. Lett. 55, 2071 (1985).

[35] H. C. Casey, D. D. Sell, and K. W. Wecht, Concentration dependence of the absorption coefficient for $n$ - and $p$-type GaAs between 1.3 and 1.6 eV, J. Appl. Phys. 46, 250 (1975).

[36] C. Chen et al., Distinguishing attosecond electron-electron scattering and screening in transition metals, Proc. Natl. Acad. Sci. U.S.A. 114, E5300 (2017).

[37] J. M. Richter, F. Branchi, F. V. de Almeida Camargo, B. Zhao, R. H. Friend, G. Cerullo, and F. Deschler, Ultrafast carrier thermalization in lead iodide perovskite probed with two-dimensional electronic spectroscopy, Nat. Commun. 8, 376 (2017). 